\title{
LEAVES WITHOUT HOLONOMY
}

\author{
D. B. A. EPSTEIN, K. C. MILLETT AND D. TISCHLER
}

We prove the following result.

THEOREM. Let $M$ be a paracompact manifold with a foliation of codimension $k$. Let $T$ be the union of all leaves with trivial holonomy. Then $T$ is a dense $G_{\delta}$ in $M$.

This result is also due independently to G. Hector [3], who has shown how useful it can be in understanding the geometry of certain foliated manifolds. In such applications one sometimes needs a form of this theorem which applies to foliated subspaces, for example a minimal subset of a foliation. In fact our proof goes through unaltered in the situation where $M$ is a locally compact, paracompact, Hausdorff foliated space such that each plaque is locally connected. We do not need to assume that $M$ is a manifold. (We recall that locally compact Hausdorff spaces satisfy the Baire category theorem.) Our treatment of the result differs from that of Hector in several respects. Firstly we give complete details of the proof. Secondly we allow the manifold which is foliated to be non-compact. Thirdly we do not restrict the differentiability class of the foliation.

Later we will give an example to show that $T$ may be empty if $M$ is not paracompact. We note that if $M$ is a paracompact manifold, then the interior of $T$ may e empty, and we will give an example which displays this behaviour.

Proof of the theorem. Let $I$ be an indexing set for a family of admissible charts $h_{i}: P_{i} \times Q_{i} \rightarrow M(i \in I)$. Let $U_{i}$ be the image of $h_{i}$. We suppose that $U_{i}$ is an open subset of $M$. In the case of a foliated manifold we suppose that $P_{i}$ is an open disk in $R^{n-k}$ and $Q_{i}$ is an open disk in $R^{k}$. In the more general situation of a foliated space, we suppose only that $P_{i}$ is a connected and open subspace of some locally compact, locally connected, Hausdorff space $P$ and that $Q_{i}$ is an open subspace of some locally compact Hausdorff space $Q$.

The foliation condition is that given $i, r \in I$ we have (locally) maps $f_{i r}$ and $g_{i r}$ such that $g_{\text {ir }}$ is one-to-one and

$$
h_{i}^{-1} h_{r}(x, y)=\left(f_{i r}(x, y), g_{i r}(y)\right)
$$

where $(x, y) \in h_{r}^{-1}\left(U_{i} \cap U_{r}\right)=h_{r}^{-1} U_{i}$. The maps $g_{i r}$ are used to define the holonomy. For our purposes the word "locally" in the above definition is an embarrassment. This word means that given $\left(x_{0}, y_{0}\right) \in h_{r}{ }^{-1} U_{i}$, there exists a small neighbourhood of $\left(x_{0}, y_{0}\right)$ and maps $f_{\text {ir }}$ and $g_{\text {ir }}$ defined on this neighbourhood, such that the above formula holds on the neighbourhood. Therefore there may correspond to a fixed pair $i, r \in I$ many different holonomy maps $g_{i r}$.

To see the difficulty more clearly we give an example where $U_{i} \subset U_{r}$. Let $R^{3}$ be

Received 21 February, 1977.

This paper is the result of conversations between the authors which occurred during the Symposium on Foliations 1975/76 at the University of Warwick, supported by the Science Research Council,

[J. LoNdON Math. Soc. (2), 16 (1977), 548-552] 
foliated by vertical lines. Let $A$ be a smoothly embedded 2-dimensional ribbon, transverse to the foliation and almost horizontal, but such that projection onto a horizontal plane is not $1-1$ on $A$. For example, the projection of $A$ could be a thickened figure of eight in the plane. If we now thicken $A$ in the vertical direction, we obtain an admissible chart in an obvious way. But there is no single holonomy map going from an open subset of $R^{2}$ to $A$.

This difficulty is dealt with in the following technical lemma, which appeared implicitly in [1]. In view of the fact that it is the kind of technical result which is often needed in foliation theory, it seems worthwhile to give complete details here.

LEMMA. Let $M$ be a Hausdorff, locally compact, paracompact, foliated space with locally connected plaques. Let $h_{i}: P_{i} \times Q_{i} \rightarrow M(i \in I)$ be a family of charts covering $M$ such that each $P_{i}$ is connected. Then there is a family of charts

$$
h_{j}: P_{j} \times Q_{j} \rightarrow M \quad(j \in J, I \cap J=\varnothing)
$$

and a map $0: J \rightarrow I$ with the following properties:

(i) $P_{j}$ is connected.

(ii) $\bar{P}_{j}$ is a compact subspace of $P_{\theta j}$ and $\bar{Q}_{j}$ is a compact subspace of $Q_{\theta j}$.

(iii) $h_{j}$ is the restriction of $h_{\theta j}$.

(iv) Writing $V_{j}=\operatorname{im} h_{j}$ and $U_{i}=\operatorname{im} h_{i}$, we have $\left\{V_{j}\right\}_{j \in J}$ is a star refinement of $\left\{U_{i}\right\}_{i \in I}$. That is to say, if $V_{j} \cap V_{k} \neq \varnothing$, then $\bar{V}_{j} \subset U_{\theta k}$.

(v) $\left\{V_{j}\right\}_{j \in J}$ is a locally finite family and each $\nabla_{j}$ is compact.

(vi) For $j, k \in J$, let $A_{j k}=\pi_{2} h_{j}{ }^{-1}\left(V_{j} \cap V_{k}\right) \subset Q_{j}$. Then there exists a unique map $g_{j k}: A_{k j} \rightarrow A_{j k}$ such that $\pi_{2} h_{j}^{-1} h_{k}=g_{j k} \pi_{2}$ on $h_{k}^{-1}\left(V_{j} \cap V_{k}\right)$. Further, $g_{j k}$ is a homeomorphism onto.

In fact we will show that if a family of charts $\left\{h_{j}: j \in J\right\}$ satisfies (i)-(v) above, then (vi) is automatically satisfied.

Proof. Let $\left\{W_{k}\right\}_{k \in K}$ be a star-refinement of $\left\{U_{i}\right\}_{i \in J}$, associated with a mapping $\psi: K \rightarrow I$. We may assume that $\left\{W_{k}\right\}$ is locally finite and that each $\bar{W}_{k}$ is compact. We shrink the covering $\left\{W_{k}\right\}_{k \in K}$ to an open covering $\left\{W_{k}{ }^{\prime}\right\}_{k \in K}$ such that $\bar{W}_{k}{ }^{\prime} \subset W_{k}$ for each $k \in K$. For each $k \in K$, we now take a finite covering of $\bar{W}_{k}^{\prime}$ by charts

$$
h_{k, r}: P_{k, r} \times Q_{k, r} \rightarrow W_{k},
$$

where $P_{k, r}$ is a connected open subspace of $P_{\psi k}, Q_{k, r}$ is an open subspace of $Q_{\psi k}$ and $h_{k, r}$ is the restriction of $h_{\psi k}$. Clearly this gives us (i) $-(v)$.

Now let $j, k \in J$ and let $y \in A_{j k}=\pi_{2} h_{j}^{-1}\left(V_{j} \cap V_{k}\right)$. Then $h_{j}\left(P_{j} \times y\right) \cap V_{k} \neq \varnothing$. It follows that $h_{j}\left(P_{j} \times y\right) \subset U_{\theta k}$. Since $P_{j}$ is connected and the inverse image under $h_{j}$ of a plaque of $U_{\theta k}$ is an open subset of $P_{j} \times y$ by the foliation condition, we see that $h_{j}\left(P_{j} \times y\right)$ lies in a single plaque of $U_{\theta k}$. We can therefore write

$$
g_{k j}(y)=\pi_{2} h_{\theta k}{ }^{-1} h_{j}\left(P_{j} \times y\right) \text { for } y \in A_{j k} \text {. }
$$

By condition (iii) of the lemma, we have

$$
g_{k j} \pi_{2}=\pi_{2} h_{k}^{-1} h_{j} \text { on } h_{j}^{-1}\left(V_{j} \cap V_{k}\right)
$$


We can write this in the form $h_{k}^{-1} h_{j}(x, y)=\left(x^{\prime}, g_{k j} y\right)$, where $x^{\prime}$ depends on $x$ and $y$. It follows immediately that $g_{k j}$ is continuous.

We show that $g_{k j}$ is a homeomorphism by proving that $g_{j k}$ is its inverse. If $y \in A_{j k}$, there exists $x$ such that $(x, y) \in h_{j}^{-1}\left(V_{j} \cap V_{k}\right)$. Then

$$
\begin{aligned}
(x, y) & =\left(h_{j}^{-1} h_{k}\right)\left(h_{k}^{-1} h_{j}\right)(x, y)=\left(h_{j}^{-1} h_{k}\right)\left(x^{\prime}, g_{k j} y\right) \\
& =\left(x^{\prime \prime}, g_{j k} g_{k j} y\right) .
\end{aligned}
$$

This completes the proof of the lemma.

Now we fix a covering by admissible charts as given by the lemma. We define an equivalence relation on $M$ by saying that $z$ is equivalent to $w$ if there is a finite chain $V_{j(1)}, \ldots, V_{j(n)}$ with $z \in V_{j(1)}, w \in V_{j(n)}$ and $V_{j(i-1)} \cap V_{j(i)} \neq \varnothing$. Each equivalence class is both open and closed. So there is no loss of generality in assuming that we have only one equivalence class. Since each $V_{i}$ only meets a finite number of other sets $V_{j}(i, j \in J)$, we see that the indexing set $J$ is countable.

Let $c$ be a periodic function of the integers into $J$ (that is, for some integer $n>0$, $c(i+n)=c(i))$, such that $V_{c(i)} \cap V_{c(i+1)} \neq \varnothing$ for any $i$. There are at most countably many such functions $c$. Any such $c$ gives rise to a holonomy map

$$
g_{c}=g_{c(0) c(1)} g_{c(1) c(2)} \ldots g_{c(n-1) c(n)} \text {. }
$$

As usual, composition of maps, where domain and range do not match, is defined by restricting to the largest possible domain and range so that they do match.

Let $z \in V_{j} \subseteq M$ and let $h_{j}: P_{j} \times Q_{j} \rightarrow V_{j}$ be the corresponding admissible chart. Let $q=\pi_{2} h_{j}^{-1} z$. One way to define the holonomy group of the leaf through $z$ at the point $z$ is as follows. We take the group of germs of homeomorphisms from a neighbourhood of $q$ in $Q_{j}$ to a neighbourhood of $q$ in $Q_{j}$, induced by $g_{c}$ for some $c$ as above, where $c(0)=j$ and $g_{c}(q)=q$.

Let the domain of $g_{c}$ be $D_{c}$ and let its range be $R_{c}$. Then $D_{c}$ and $R_{c}$ are open subsets of $Q$. Let $F_{c}$ be the fixed point set of $g_{c}$. That is,

$$
F_{c}=\left\{x \in Q: x \in D_{c} \cap R_{c} \text { and } g_{c}(x)=x\right\} \text {. }
$$

$F_{c}$ is closed in $D_{c}$ and also in $R_{c}$. Let $B_{c}$ be boundary of $F_{c}$ in $Q$. Then $B_{c}$ has void interior. Let $B$ be the union of all the $B_{c}$ (note that this is a countable union).

The next observation is that if $y \in Q_{j} \backslash B$ and $x \in P_{j}$ then $h_{j}(x, y)$ lies on a leaf with trivial holonomy. Suppose not. Choose a loop on the leaf along which the holonomy is not trivial. Let $V_{c(0)}, V_{c(1)}, \ldots, V_{c(n)}$ be any chain covering this loop such that $c(n)=c(0)$ and such that the loop can be cut up into $n$ intervals, with the $i$ th interval lying in $V_{c(i)}$. Then the holonomy along the loop is given by $g_{c}$. Moreover, the initial point of the loop corresponds to a fixed point $y_{0}$ of $g_{c}$. Now $y_{0}$ is not in the boundary of the fixed point set of $g_{c}$. Therefore $y_{0}$ has a neighbourhood $N$ in $Q$ which is entirely contained in the fixed point set of $g_{c}$. But then $g_{c}$ is fixed on $N$ so that the holonomy induced at $y_{0}$ by the loop is trivial.

Finally, let $\left\{W_{j}\right\}_{j \in J}$ be an open covering of $M$ with $\bar{W}_{j} \subset V_{j}$. Then

$$
\bar{W}_{j} \cap h_{j}\left(P_{j} \times\left(B_{c} \cap Q_{j}\right)\right)
$$

is a closed subset of $M$ with empty interior. As $j \in J$ and $c$ vary we obtain a countable collection of closed sets with void interior. Their complement $T$ in $M$ is a dense $G_{\delta}$. By the preceding paragraph, $T$ consists of points with trivial holonomy. 
Examples: To observe that $T$ may have empty interior we construct the following example. Let $\alpha: T^{2} \rightarrow T^{2}$ be the diffeomorphism given by the linear map $\tilde{\alpha}: R^{2} \rightarrow R^{2}$ with $\tilde{\alpha}=\left(\begin{array}{ll}2 & 1 \\ 1 & 1\end{array}\right)$. The linear map $\tilde{\alpha}$ preserves two foliations of $R^{2}$, namely the straight lines parallel to one of the eigenvectors. Let $F_{1}$ and $F_{2}$ be the foliations of $T^{2}$ which result from projecting down to $T^{2}$ the two invariant foliations of $R^{2}$. Let $M$ be the mapping torus of $\alpha$. That is to say, $M$ is a 3-dimensional manifold obtained from $T^{2} \times R$ by identifying $(x, t)$ with $(\alpha x, t+1)$ for each $x \in T^{2}$ and $t \in R$. Then $M$ has a codimension-one foliation $G$ which is a projection to $M$ of the foliation $F_{1} \times R$ of $T^{2} \times R$. It is well known that the periodic points of $\alpha$ are a dense subset of $T^{2}$. A leaf of $G$ passing through $(x, 0)$, where $x$ is periodic, is easily seen to have non-trivial holonomy and the union of such leaves is dense.

We conclude by describing a modification of an example of Milnor [2] to give a codimension-one foliation of a non-paracompact, Hausdorff 3-dimensional manifold which has only one leaf, and that leaf has holonomy. The foliated manifold has charts $\left\{U_{\alpha}, h_{\alpha}\right\}_{\alpha \in R}$ with $h_{\alpha}$ a homeomorphism of $R^{3}$ onto $U_{\alpha}$ such that

if and only if

$$
h_{\alpha}\left(x_{\alpha}, y_{\alpha}, z_{\alpha}\right)=h_{\beta}\left(x_{\beta}, y_{\beta}, z_{\beta}\right), \alpha \neq \beta,
$$

(i) $x_{\beta}=x_{\alpha} \neq 0$ (this common value is denoted by $x$ ),

(ii) $y_{\beta}=y_{\alpha}+(\alpha-\beta) / x$,

(iii) $z_{\beta}= \begin{cases}z_{\alpha}+(\alpha-\beta), & x>0 \\ 2^{\beta-\alpha} z_{\alpha}, & x<0 .\end{cases}$

We note that the associated changes of co-ordinates preserve the planes $z=$ constant, $x>0$ and $z=$ constant, $x<0$ so that $M$ has a codimension-one foliation given by the condition $z=$ constant. The associated holonomy maps are

$$
\begin{aligned}
\text { (i) } x>0 & g_{\beta \alpha}^{+}\left(z_{\alpha}\right)=z_{\alpha}+(\alpha-\beta), \\
\text { (ii) } x<0 & g_{\beta \alpha}^{-}\left(z_{\alpha}\right)=2^{\beta-\alpha} z_{\alpha} .
\end{aligned}
$$

To see that there is only one leaf, note that

$$
g_{0, z} g_{z, 0}^{+}(\omega)=2^{-z}(\omega-z) \text {. }
$$

Therefore in the chart with $\alpha=0$, the leaf corresponding to $\omega=z$ is the same as the leaf corresponding to $z=0$. This leaf has non-trivial holonomy since if $z \neq 0$, the above map has $z /\left(1-2^{z}\right)$ as a fixed point, and the derivative of $g_{0, z}^{-} g_{z, 0}^{+}$at this fixed point is $2^{-z}$.

Remark. The easiest way to prove that the above manifold is Hausdorff is to use the continuous map to $\mathbb{R}^{2}$ which maps $\left(x_{\alpha}, y_{\alpha}, z_{\alpha}\right)$ to $\left(x_{\alpha}, x_{\alpha} y_{\alpha}+\alpha\right)$. This is consistent with the equivalence relation defining the manifold. Now use the fact that $\mathbb{R}^{2}$ is Hausdorff. 


\section{References}

1. D. B. A. Epstein, " Foliations with all leaves compact", Annales de l'Institut Fourier, 26 (1976), 265-282.

2. J. Milnor, "Foliations and foliated vector bundles", mimeographed notes, MIT, 1969.

3. G. Hector, "Feuilletages en cylindres", (to appear).

D. B. A. Epstein, University of Warwick, Coventry CV4 7AL, England.

K. C. Millett, University of California, Santa Barbara CA93106, U.S.A.

D. Tischler, Queens College, (CUNY),

Flushing, N.Y. 11367, U.S.A. 